\title{
Tsafon
}

Revue d'études juives du Nord

$81 \mid 2021$

Des synagogues à travers les âges Lieux de prières, lieux d'études et autres fonctions

\section{Hadas-Lebel Mireille, Les Pharisiens dans les Évangiles et dans l'Histoire}

\section{Olivier Rota}

\section{(2) OpenEdition \\ Journals}

Édition électronique

URL : https://journals.openedition.org/tsafon/3988

DOI : $10.4000 /$ tsafon.3988

ISSN : 2609-6420

Éditeur

Association Jean-Marie Delmaire

Édition imprimée

Date de publication : 1 juillet 2021

Pagination : 157

ISSN : 1149-6630

Référence électronique

Olivier Rota, «Hadas-Lebel Mireille, Les Pharisiens dans les Évangiles et dans l'Histoire », Tsafon [En ligne], 81 | 2021, mis en ligne le 01 juillet 2021, consulté le 15 septembre 2021. URL : http:// journals.openedition.org/tsafon/3988; DOI : https://doi.org/10.4000/tsafon.3988 


\section{À travers les livres ......}

Hadas-Lebel Mireille, Les Pharisiens dans les Évangiles et dans l'Histoire, Paris, Albin Michel, 2021, 204 p., 19,90€.

Qui sont les Pharisiens ? Les Évangiles, on le sait, ont durablement associé le nom de ce parti à l'idée d'hypocrisie et d'ostentation dans les dévotions, tant et si bien que toute recherche sur des Pharisiens doit commencer par percer un voile de préjugés. C'est ce travail qu'entreprend l'étude de Mireille Hadas-Lebel. Les qualités de clarté d'exposition de l'auteur sont connues. MHL présente et étudie les sources disponibles: Flavius Joseph, les Évangiles, la Mishna, les Midrashim tannaïtiques. Chacune de ces sources est restituée dans son contexte d'écriture, de telle manière que l'évolution de ce contexte explique leurs différences de contenu et d'orientation. L'attention portée sur ces sources permet de mettre en évidence ce que furent les Pharisiens : un groupe de Juifs pieux, mais pas obligatoirement piétistes, dont l'existence est attestée à partir des débuts de la dynastie hasmonéenne et qui se distingue et s'oppose aux Saducéens sur la question de l'application de la Torah, notamment sur la possibilité d'une résurrection.

Tout l'intérêt de l'ouvrage se situe dans la manière dont MHL présente la diversité du judaïsme du $\mathrm{I}^{\mathrm{er}}$ siècle et y inscrit le christianisme. L'auteur montre que les questions posées par les Pharisiens à Jésus sont loin d'être des questions-pièges : ce sont pour partie des questions en débat à l'époque entre les Juifs. Tout comme les Pharisiens, Jésus apparaît préoccupé de pureté ; le portrait que dresse MHL des Pharisiens rapproche Jésus de ce parti. Se détachant de l'Évangile de Mathieu, MHL souligne les passages de l'Évangile de Luc où les Pharisiens approuvent Jésus. Le mépris des Pharisiens, lorsqu'il s'exprime, relève plutôt de celui qu'ils ont pour les 'amei ha-aretz, les gens du peuple. De fait, Jésus a raisonnablement pu évoluer dans le milieu des Pharisiens, tant son enseignement ne prend sens que dans la pensée de ce groupe. Jésus se démarque des Pharisiens plus qu'il s'y oppose. La querelle entre Jésus et les Pharisiens relèverait ainsi de la "querelle de famille », thèse déjà soutenue par d'autres chercheurs. Aussi, la rupture entre judaïsme et christianisme ne s'expliquerait vraiment que par les conditions nées de la destruction du Temple en 70 , et de la nécessité pour le judaïsme de supprimer toute dissidence.

Olivier Rota

Schumacher-Brunhes Marie (coordinatrice), «Entre deux mondes : Marges, passage et modernité dans la culture yiddish au XX ${ }^{\text {ème }}$ siècle », Germanica n $^{\circ} 67,2020,249$ p., $18 €$.

Le dossier du numéro 67 de Germanica est consacré à la manière dont les communautés juives dans les métropoles européennes et nord-américaines ont vécu et ont contribué à la construction de l'espace urbain moderne. Douze contributions y sont réunies, dix en 
français et deux en allemand. Dans son avant-propos, Marie Schumacher-Brunhes invite les lecteurs à se pencher sur les principales villes juives telles que Varsovie, Vienne, New York ou Montréal, comme des lieux de transit ${ }^{1}$, des $«$ espaces autres $»^{2}$ et des tiersespaces $^{3}$ où les contrastes s'estompent. Ce dossier se connecte ainsi à l'étude de l'hybridité culturelle où l'on voit la culture yiddish influencée par l'existence des cultures qui l'entourent et la création de lieux de transition entre l'ancien et le nouveau, le traditionnel et le moderne, le juif et le non-juif, jusqu'à ce que la bipolarité radicale cesse d'exister.

Dans ce contexte, l'article de Jean Baumgarten parle de la multiplication de nouveaux espaces propices à l'expression d'une dissidence et l'émergence, avant même les premiers soubresauts de la sécularisation, d'espaces des marges venant dérégler l'espace encadré de la kehilah (communauté juive instituée). Arnaud Bikard évoque dans sa contribution l'Union des Écrivains et Journalistes juifs à Varsovie, mieux connue sous le nom de Tłomackie 13, en référence à l'adresse qu'elle occupait, et les mythes qui y furent associés. Ceux-ci relayent une hybridité foncière entre le shtetl familier et les aspirations culturelles modernes, qui s'exprimait aussi dans les luttes qui s'y déployèrent pour obtenir une domination symbolique.

Dans le champ musical et plus précisément celui de l'art cantorial, Jeremiah Lockwood observe le positionnement des chantres de synagogue opposés à la commercialisation de la musique sacrée que permettent les concerts mais aussi la nouvelle technologie de l'enregistrement sur disque. L'auteur réalise une étude de cas à partir de l'agenda musical de 1924 du chantre Elias Zaludkovsky (1888-1943) pour analyser l'affranchissement de la musique sacrée de son contexte rituel, qui génère un tiersespace de culture liturgique.

Dans le domaine littéraire, Michèle Tauber met en exergue les espaces qualifiés d'« autres » par Foucault tels qu'ils sont évoqués par l'écrivain hébraïque David Vogel (1891-1944) dans son roman La vie conjugale. Vogel y décrit la vie de jeunes immigrants juifs d'Europe de l'Est essayant de trouver leur place dans la société viennoise au début des années vingt. L'auteur se concentre d'abord sur l'analyse de la description des lieux physiques de la ville tels que le café viennois, l'hôpital psychiatrique ou l'asile pour les pauvres comme tiers-espaces avant de souligner que le tiers-espace est représenté aussi par la langue : l'hébreu et le yiddish. Delphine Bechtel choisit également d'examiner l'espace des cafés, cette fois dans la ville de Lemberg entre les deux guerres, un lieu mixte, fréquenté par des juifs et des non-juifs, multilingue, qui témoigne des interrelations entre les différentes cultures et de la place des entrepreneurs juifs comme médiateurs entre la haute et la basse culture.

Une partie du dossier est dédiée à la poétesse yiddish Debora Vogel (1900-1942). Marie Schumacher-Brunhes décrit tout d'abord la vie de cette femme, une intellectuelle juive se déplaçant entre les grandes villes d'Occident, qui devient une représentante de l'avant-garde de la culture yiddish et polonaise. Après avoir appris le yiddish à l'âge adulte, elle a fait le choix radical d'écrire dans cette langue. Ainsi a-t-elle publié deux recueils de poésie en yiddish à partir desquels ont été rassemblés 17 poèmes pour ce dossier, Tog-figurn. Lider (Figures du jour. Poèmes; 1930) et Manekinen (Mannequins ; 1934), pour lesquels Batia Baum livre des traductions inédites. Dans ces recueils, dans lesquels la ville de Lemberg sert par ailleurs souvent de décor, Debora

\footnotetext{
${ }^{1}$ Victor Turner, The Ritual Process: Structure and Anti-Structure, Ithaca, New York, Cornell University Press, 1996.

${ }^{2}$ Michel Foucault, «Des espaces autres », dans Dits et écrits II, Paris, Gallimard, 2000 [dans Architecture, mouvement, continuité, Paris, 1984], p. 1571-1581.

${ }^{3}$ Edward W. Soja, Thirdspace. Journeys to Los Angeles and Other Real and Imagined Places, Oxford, UK, Blackwell, 1996 ; Homi K. Bhabha, The Location of Culture, London, Routledge, 1994.
} 
Vogel évoque l'aliénation créée par la modernité et l'industrialisation ainsi que la triste réalité de la condition féminine.

L'article de Cécile Rousselet est quant à lui consacré à l'auteur Esther Kreitman (18911954) et à son roman de 1944 Brilyantn (paru en français sous le titre Le Diamantaire). Ce texte suit les tribulations d'une famille juive entre Anvers et Londres au moment du déclenchement de la Première Guerre mondiale, partagée entre le monde familier du shtetl qu'elle a laissé derrière elle et le monde antagonique de la spéculation financière représenté par le commerce des diamants. Cécile Rousselet décrit la langue yiddish au cœur de l'intrusion de voix multiples dans l'espace de la ville qui devient un tiersespace. Valentina Fedchenko analyse également le rôle joué par les espaces tiers, cette fois à partir des œuvres d'Isaac Bashevis Singer (1904-1991) et montre que celles-ci produisent une culture alternative dans un espace situé entre les traditions littéraires juives et américaines permettant une troisième couche d'interprétation. Le dernier article en langue française dans ce dossier, rédigé par Chantal Ringuet, porte sur l'image de la forêt comme tiers-espace dans la poésie en yiddish de Rachel Korn (1898-1982). Chantal Ringuet décrit l'influence des paysages du Canada sur la poétesse immigrée et son lien intime avec les arbres et les montagnes, tout en évoquant un Yiddishland perdu. Les articles en allemand se concentrent directement ou indirectement sur la ville de Berlin. Astrid Starck-Adler décrit la ville de Berlin comme un lieu de transit, au début $\mathrm{du} \mathrm{XX}^{\text {ème }}$ siècle, pour les juifs immigrés venus d'Orient sur leur chemin vers l'Amérique. Berlin était un lieu de rencontre avec l'Occident « culturel » où la littérature yiddish a pu aborder de nouveaux espaces de transition temporaires, valorisant les points de contact entre l'Est et l'Ouest. Elke-Vera Kotowski donne quant à elle un aperçu de l'essor des traductions vers le yiddish de la littérature en langue allemande jusqu'au début de la Seconde Guerre mondiale, surtout à Berlin, devenu provisoirement l'un des principaux sites de publication.

Ce dossier, riche et étoffé, permet de mieux appréhender la culture yiddish au $\mathrm{XX}^{\mathrm{e}}$ siècle comme un moment de passage entre deux mondes. Nous en recommandons la lecture.

Ilanit Ben Dor-Derimian

Savy Pierre (sous la direction de), Histoire des Juifs. Un voyage en 80 dates de l'Antiquité à nos jours, Paris, Presses universitaires de France, sept. 2020, 586 p., $29 €$.

En collaboration avec Katell Berthelot (directrice de recherche au CNRS et spécialiste du judaïsme de l'époque hellénistique et romaine) et Audrey Kichelewski (maîtresse de conférences en histoire contemporaine à l'université de Strasbourg et membre junior de l'Institut universitaire de France), Pierre Savy (directeur des études pour le Moyen Âge à l'École française de Rome) publie un ensemble de 80 textes sur l'histoire des Juifs dans le monde et couvrant pas moins de 32 siècles (de 1207 av notre ère avec la stèle de Mérenptah à la prise d'otages de l'Hyper Cacher en 2015) : une gageure ! Les choix ont dû susciter bien des discussions et nécessairement le lecteur pourra toujours déplorer un, voire plusieurs oublis.

Lors d'une visio conférence pour présenter l'ouvrage, P. Savy se défendait de vouloir écrire une histoire lacrymale et déclarait retenir aussi des périodes et des événements heureux de ce passé juif. Il assumait aussi des absences comme Masssada, la Nuit de Cristal, plus regrettable Qumran ou le procès de Nuremberg ou encore Ben Yehouda. En revanche, le lecteur aura plaisir à découvrir des faits moins connus comme la date de 212 lorsque les Juifs deviennent des citoyens romains, ou des personnages restés trop longtemps dans l'ombre comme la publication des poèmes de Debora Corcos Ascarelli, une première pour une poétesse, et la nomination de la première femme rabbin en 1935. 
Très souvent une date est le prétexte pour développer une histoire plus longue. Par exemple, l'article de Perrine Simon-Nahum qui présente la fondation de la Wissenschaft des Judentums prolonge l'étude jusqu'à la fin du $\mathrm{XIX}^{\mathrm{e}}$ siècle avec la Science du Judaïsme en France. De même Cyril Grange informe sur la dynastie des Rothschild à partir de la date du 29 septembre 1822, jour où « l'empereur d'Autriche François I ${ }^{\text {er }}$ élève au rang de baron les cinq fils de Mayer Amschel Rothschild ». L'affaire de Simon de Trente, exposée par Pierre Savy, permet d'écrire une brève histoire des meurtres rituels.

Les notices sont confiées à des spécialistes du sujet traité comme Christian Ingrao sur la conférence de Wannsee ou Catherine Nicault sur la déclaration Balfour, ou encore Thomas Römer sur le début de l'empire perse pour la date de 539 avant notre ère et Dominique Bourel pour un texte sur Moses Mandelssohn. Les trois directeurs de l'ouvrage ont réussi à rassembler 70 auteurs pour ces 80 dates.

On l'aura compris, l'œuvre est à consulter à la manière d'une encyclopédie, bien que réduite. Il faut saluer les outils de fin de pages : les trois index très utiles, des noms, des lieux et des matières. Et chaque article est suivi d'une brève bibliographie.

Une question se pose : qu'est-ce qui différencie cet ouvrage de l'Histoire universelle des Juifs dirigée par Élie Barnavi (1992 et 2002) ? Celui de Barnavi se présente davantage comme une sorte d'atlas avec une iconographie bien plus abondante pour chaque thème traité sur une double page. Ici les auteurs développent plus longuement un sujet, environ cinq à six pages, les différentes notices sont de longueur à peu près uniforme, et les illustrations comme les cartes sont rares.

Malgré ses faiblesses inhérentes au choix des directeurs, cette histoire de Juifs en 80 dates doit figurer dans les bibliothèques des curieux de cette histoire, des instituts et des départements d'université spécialisés dans les études juives.

Danielle Delmaire

Keller-Rosenberg Lili, Et nous sommes revenus seuls, Paris, Plon, avril 2021, 157 p., $15 €$.

Plus connue sous son nom d'épouse, Lili Leignel signe, chez Plon et avec la participation de Virginie Carton, son second livre sur sa déportation à Ravensbrück puis Bergen-Belsen, avec sa maman et ses deux frères : les enfants avaient respectivement onze ans, dix ans et trois ans au moment de leur arrestation, en octobre 1943, à Roubaix dans le Nord. Comme pour son livre précédent, elle adopte prioritairement le nom de sa maman, Charlotte Keller, épouse Rosenberg. Bel hommage rendu à cette mère de famille qui lutta avec une force et un courage exceptionnels pour que vivent, survivent ses trois jeunes enfants dans un univers concentrationnaire inhumain.

Inlassablement, Lili Leignel accomplit la «mission» qu'elle s'est donnée, dès lors qu'elle apprit que l'on était capable de nier la réalité de la Shoah : témoigner auprès des enfants et des adolescents de ce qu'elle et ses frères ont subi comme souffrances physiques et morales parce qu'ils étaient juifs. Elle a commencé à se rendre dans les écoles, collèges et lycées au début des années 1980, dans sa ville de Lille d'abord, dans la région du Nord ensuite et finalement partout en France et même en Allemagne. Tsafon a pu recueillir un premier récit et l'a publié dans son $\mathrm{n}^{\circ}$ 9-10 de l'été-automne 1992, p. 28-37. Plus récemment, des enseignants du collège Prévert à Houdain (Pas-deCalais) ont à leur tour publié, fin 2017, ses souvenirs augmentés des lettres d'élèves reçues, en grand nombre, par Lili Leignel après ses passages dans leurs établissements. Pour les éditions Plon, elle s'en tient au seul récit de sa déportation. Mais l'approche n'est plus la même. Alors qu'auparavant, elle mettait en avant les difficultés physiques, le titre du précédent ouvrage en témoigne : Moi, Lili Keller-Rosenberg, je suis encore 
là, rescapée de la Shoah et passeur de mémoire, ici, avec la présentation de ce qu'elle appelle sa "mission», elle s'arrête davantage sur les souffrances morales : Et nous sommes revenus seuls!, « revenus seuls » des camps abominables sans la maman, trop malade pour quitter Bergen-Belsen à l'ouverture de ce camp. Les enfants débarquent « seuls » à l'hôtel Lutétia, ignorant tout du sort du papa qui n'a pas survécu à Buchenwald.

Le récit débute donc par la fin, avec l'évacuation de Bergen-Belsen et la séparation douloureuse d'avec la maman inconsciente car atteinte de la phase la plus grave du typhus. « Nous restons seuls, désemparés. Livrés à nous-même » (p. 15) et plus loin «Nous sommes les seuls enfants de ce convoi de retour» (p. 17). Lors d'une étape à Bruxelles : «Et nous sommes là, tous les trois collés comme un seul bloc, étourdis dans cette foule inconnue » venue les accueillir (p. 19); «[...] mais personne n'est là pour nous. Personne n'est venu nous chercher» (p. 20). Ce n'est qu'après ces premières pages du retour traumatisant et peu libérateur que Lili Leignel reprend son récit de la vie familiale heureuse avant la guerre, l'arrestation en octobre 1943, de toute la famille, l'internement à la prison de Loos, près de Lille, puis à Saint-Gilles (Bruxelles), le séjour humiliant et dépravant à la caserne Dossin de Malines d'où partaient les convois de Juifs et de Tsiganes de Belgique et du nord de la France, vers Auschwitz.

C'est alors la séparation d'avec le père qui est dirigé, comme tous les hommes, vers Buchenwald tandis que les femmes et les enfants prennent la direction de Ravensbrück où la maman et les trois enfants côtoient des résistantes au caractère trempé (Geneviève de Gaulle, Martha Desrumaux entre autres), qui leur accordent une grande attention et aident la maman dans ses efforts à maintenir ses enfants en vie. Bergen-Belsen est également décrit dans toute son horreur: abondance des épidémies, monceaux de cadavres, la faim, la peur et la maman gravement malade, agonisante.

Dans les quarante dernières pages, Lili Leignel revient sur la solitude du retour puis le retour à la vie familiale avec la maman rentrée presque guérie mais sans le papa. Elle termine en persistant à évoquer sa «mission » : témoigner. "Et quand je regarde le chemin parcouru, les dizaines de milliers de jeunes croisés sur ma route avec lesquels j'ai pu échanger, auxquels j'ai pu transmettre mon témoignage, mon histoire, ma leçon de citoyenneté, de tolérance et d'humanisme, je crois que je peux commencer à affirmer: 'mission accomplie'» sont ses derniers mots. Car Lili Leignel refuse une évocation victimaire et larmoyante de son passé : de ce récit il faut tirer des leçons dont tiendront compte les adultes de demain.

Danielle Delmaire

Lévy-Garber Ode, Passé sous silence, réédition des entretiens d'Ode Lévy-Garber réalisés par Jean-Paul Woodall (bruits du temps) en 2015, version augmentée en documents et annexes, Nice, octobre 2020, 248 p., prix non indiqué.

L'équivoque du titre, selon que le mot "passé» est compris comme participe ou comme substantif, persiste après la lecture. En effet, faut-il comprendre que le récit a été peu entendu donc est passé (participe) sous silence ou bien que la difficulté de parler après la guerre a occasionné un silence et ce passé (substantif) surgit bien tardivement ? Peu importe car finalement les deux options peuvent accompagner la lecture du récit et la découverte du passé.

Odette Lévy, épouse Garber, est née en 1931, après quatre autres enfants, dans une famille juive originaire de l'Empire ottoman. C'est avant la Première Guerre mondiale que la famille de son père quitte un pays où l'antisémitisme et l'insécurité sont menaçants, pour divers pays : la Palestine ottomane, le Brésil et la France. Le couple s'est installé à Lille où la vie s'écoule sans encombre jusqu'au décès de la maman en 
1938. Joseph Lévy se trouve veuf, jeune encore - il a un plus de quarante ans - avec quatre enfants adolescents et la petite dernière qui a tout juste sept ans.

Â la déclaration de la guerre, le père emmène toute sa famille vers le sud avec l'espoir de gagner rapidement l'Espagne et de s'embarquer pour le Brésil où un frère pourrait l'accueillir. La tentative échoue et en 1940 un nouvel exode les amène à Saint-Gaudens. La jeune Odette y mène une vie pas trop mouvementée même si l'inquiétude taraude son père. Ses souvenirs sont plutôt plaisants. La famille reste à Saint-Gaudens jusqu'à la fin de 1942.

Après ces deux années, les nuages se font plus denses et, pour s'être déclarée, il devient plus prudent pour la famille de se réfugier dans un petit village non loin de Cahors : Arcambal. Odette se fait de nouveaux amis et fréquente l'école. Joseph, le père, parvient à travailler tandis que les sœurs ainées se débrouillent pour gagner quelque argent et entretenir la maison. Les rapports avec les habitants du village sont tout à fait cordiaux et pour Odette la vie est heureuse malgré les privations. Elle est trop jeune encore pour comprendre le sens des visites d'inconnus : son père Joseph et son frère Maurice âgé de 18 ans en 1944 participent à la résistance locale. La maison devient un lieu que l'on pense sûr pour cacher des armes, des documents compromettants et pour des rencontres secrètes.

Le drame arrive le 2 juillet 1944 lorsqu'une perquisition met à jour toutes ces activités illicites et dangereuses. En l'absence de Violette, les trois sœurs, Esther, Judith et Odette sont arrêtées. Joseph et Maurice sont emmenés pour interrogatoire : Maurice est horriblement torturé et finalement fusillé tandis que son père disparaît sans que ses filles parviennent à retrouver sa trace après la guerre.

Originaires de Turquie, pays allié au Reich, les trois sœurs sont déportées à Ravensbrück par le convoi 81, parti de Toulouse le 30 juillet 1944, alors que la ville est libérée une vingtaine de jours plus tard. Odette, âgée de 13 ans, connaît les affres de la déportation mais la présence de ses sœurs la soutient et la rassure. Elle est évacuée à Bergen-Belsen où son témoignage est très proche du récit de Lili Leignel : la faim, la peur, les maladies, l'absence de toute hygiène. Nous y lisons la même souffrance physique et morale et l'enfant qui ne comprend pas ce basculement dans l'horreur.

L'après n'est pas un retour au bonheur. Les sœurs ne parlent pas du drame d'Arcambal et encore moins de la déportation. C'est Violette, alors qu'elle a échappé à l'arrestation, qui surmonte le plus difficilement le traumatisme de la disparition du père et du frère. Longtemps Odette n'a pas voulu s'exprimer : la parole n'était pas une libération car elle ravivait la douleur. Le mutisme devenait protecteur. Toutefois, pas indéfiniment puisque dans les années 2010, devenue octogénaire, elle confie ses souvenirs à Jean-Paul Woodall. Des redondances parsèment le récit mais ne perturbent pas la lecture car il est évident qu'Odette doit se répéter pour s'imprégner de ses souvenirs et pour convaincre le lecteur.

Outre le témoignage sur ce que fut la déportation à Ravensbrück et Bergen-Belsen, il faut retenir de cette lecture l'engagement résistant, fréquent chez les juifs réfugiés dans le sud de la France, des proscrits du régime. Ces juifs ne se sont pas résignés : ils ont combattu et n'ont pas trahi, même sous la torture. Ils se sont sacrifiés pour un pays qu'ils admiraient et ils ont refusé l'humiliation. C'est cela aussi et surtout cela que contient le témoignage d'Odette Lévy-Garber.

Le livre est enrichi par de nombreuses photos et documents, notamment les lettres et imprimés révélant les démarches longues et difficiles entreprises par Joseph Lévy pour obtenir la nationalité française, dans les années 1932-1933. La France, qu'il affectionnait tant, tergiversait pour l'intégrer avant de refuser de l'accepter parmi ses citoyens. Mais Joseph avait compris qu'il existait une autre France avec laquelle et pour laquelle il a combattu.

Danielle Delmaire 
Fradet Jean-Jacques, Belleville Mamie Blues, Paris, L'Harmattan, 2020, 356 p., $29 €$. Préface d'Henri Raczymow, postface de Claude Lelouch.

Jean-Jacques Fradet a rendu un bel hommage, post mortem à sa " mamie », sa grandmère maternelle : Chana Szajman née Mitzenmacher. Elle était venue de sa Pologne natale et avait épousé, à Paris et alors âgée de 19 ans, le 30 décembre 1930 Yankel Szajman originaire de Varsovie et de 17 ans son aîné. Le couple eut une petite fille Louise et Yankel fut arrêté le 7 septembre 1942, probablement sur dénonciation d'une voisine, puis déporté de Drancy le 16 septembre suivant.

Fort heureusement, les premières pages s'ouvrent sur un arbre généalogique de la famille Mitzenmacher dont il sera beaucoup question dans le livre et sur un «who's who » qui en donne l'explication. Cela permet de suivre chacun des membres de la famille au fur et à mesure de la lecture.

Le livre respire l'amour filial du petit-fils envers sa grand-mère toujours appelée « mamie » : une jeune couturière qui, dans les années qui suivirent la guerre, éleva seule ses deux filles Louise et Germaine, la mère de l'auteur. L'on devine aisément une grande complicité entre les deux personnages. Le récit familial, qui englobe toute la fratrie de «mamie » et ses descendants, se déroule sur de nombreux chapitres parfois très brefs - quelques lignes - parfois bien plus développés. Ils sont tous datés et localisés : avant, pendant et après la Shoah; en Pologne, en France et ailleurs. La chronologie n'est pas respectée : le lecteur est promené depuis 1911 jusqu'aux années 2000 selon des allers et retours qui ponctuent les souvenirs de l'auteur. En France, ces souvenirs familiaux nous entrainent principalement à Belleville, quartier juif où vécut « mamie » et ses filles - un plan en début du livre aide le lecteur - ainsi que dans le Vercors où les cousins Denner s'engagent dans le maquis et dans toutes les caches nécessaires aux juifs menacés de déportation, à Lille également où, malgré ce qu'en dit l'auteur (p. 232-233), il n'y a pas de corons mais des courées et où le beffroi de la ville n'a pas de carillon!

Le récit de la période de la guerre, bien que dispersé en divers chapitres, occupe une place importante. On y rencontre des familles juives qui déploient des ruses complexes pour survivre, parfois en vain: Yankel, le mari de " mamie » est dénoncé, l'enfant Marcel, neveu de «mamie », est extrait de sa cachette pour être déporté, " mamie » entreprend un voyage périlleux en zone libre pour revenir à Belleville. On y rencontre encore des résistants et des collaborateurs. La saga familiale entraîne aussi le lecteur en Pologne où les cousins et cousines périssent dans la Shoah.

Outre la présence des membres de la famille de «mamie », celle de son neveu Charles Denner, cousin de Germaine la mère de l'auteur, est très prégnante dans ce récit. Son profil ressemble beaucoup à celui de Germaine, sa cousine (p. 57). Une lecture attentive de l'arbre généalogique fait découvrir la question lancinante qui taraude l'auteur : qui est son grand-père maternel ? "Mamie» a accouché de Germaine deux ans après l'arrestation de son mari et la petite fille porte le nom de naissance de « mamie » et non celui de son mari. Les investigations de l'auteur nous laissent sans réponse car il ne l'a pas et ne l'a jamais eue. Il s'en tient à des présomptions à peine suggérées, au lecteur d'en tirer d'éventuelles conclusions. Et le mystère parcourt tout le livre.

Par ces souvenirs, J-J Fradet associe l'histoire familiale à l'Histoire des Juifs immigrés en France au $\mathrm{XX}^{\mathrm{e}}$ siècle et à l'Histoire de la Shoah en Europe. Le mélange des genres : histoire et journal intime peut dérouter mais n'est pas désagréable finalement. Ainsi l'on peut découvrir, en fin de volume, les bonnes recettes de cuisine que «mamie» a importées de sa Pologne natale et, au fil des pages, on peut fredonner les paroles de chants des diverses époques. 
En revanche, l'historien peut regretter que les nombreuses photos, parfois trop sombres, ne soient pas référencées et présentées plus précisément.

Les préface et postface de deux célébrités juives s'explique l'une, celle d'Henri Raczymow, par la proximité de l'auteur avec le romancier, traducteur en français d'œuvres littéraires yiddish; l'autre, celle de Claude Lelouch, par l'amitié qui liait le réalisateur et scénariste de films à l'acteur Charles Denner. En effet, dans les années 1970, ce dernier tourne dans plusieurs films de Lelouch dont Le Voyou (1970) et L'Aventure c'est l'aventure (1972). Charles Denner s'illustra également au théâtre dans la troupe du TNP de Jean Vilar puis Georges Wilson.

Danielle Delmaire

Baum Thomas et Vanden Daelen Veerle (dir. de publication), La Shoah et les droits humains, catalogue de l'exposition permanente, Gand, éditions Marot-Tijdsbeeld et Malines Kazerne Dossin, 2020, 480 pages, 680 illustrations, prix non indiqué.

Cet ouvrage est le catalogue de l'exposition permanente du musée de la Caserne Dossin à Malines dont le thème principal est la Shoah mais s'y ajoutent des réflexions sur les droits humains en général et partout dans le monde. Huit collaborateurs ont aidé les deux auteurs pour réaliser ce volumineux catalogue, en hommage à Maxime Steinberg, le premier historien belge qui a travaillé sur la déportation des Juifs de Belgique.

Le catalogue donne à voir l'essentiel de l'exposition. Il repose sur la publication d'abondantes photos d'époque et la reproduction de documents d'archives ou d'objets comme des cartes d'identité ou d'alimentation, et encore de caricatures. Les textes sont peu développés et, à part quelques pages de présentation des différentes parties du livre, ils sont réduits à la l'explication des illustrations.

L'ouvrage repose sur trois parties: les masses (une centaine de pages), l'angoisse (presque 350 pages) et la mort (un peu plus d'une centaine de pages). Ce sont donc prioritairement les conditions de survie pour contrer la persécution qui ont retenu l'attention des auteurs.

Les masses. L'exposition s'attarde sur des photos de foule: prisonniers politiques allemands des années 1930, militants nazis rassemblés dans des stades, rassemblements dans les rues des villes allemandes en soutien au dictateur ou lors des exactions contre les Juifs. En opposition d'autres photos montrent des défilés du $1^{\text {er }}$ mai organisés par des militants juifs avec des banderoles en yiddish. En parallèle avec l'actualité, une photo d'une barque surchargée de réfugiés, isolée en pleine mer, impressionne.

L'angoisse. L'abondance des mesures répressives occupe ces 350 pages. L'accumulation des ordonnances, l'enregistrement des Juifs avec les listes qui s'allongent, le marquage des entreprises et des magasins, les étoiles cousues sur les vêtements, les cartes d'identité estampillées Jood et Juif, les spoliations sont autant de sources d'angoisse pour les Juifs de Belgique durant toute la guerre. Des photos font découvrir les terribles conditions de détention au fort de Breendonk et à la caserne Dossin. S'ajoute la convocation pour un travail forcé dans les camps pour Juifs (Judenlager) du Boulonnais dans le nord de la France. Enfin les rafles et la clandestinité se superposent à toutes ces mesures génératrices d'angoisse. Les cartes de la répartition des refuges pour les Juifs sont hélas peu lisibles (p. 280-283). Cette partie centrale, et la plus importante, rassemble, sur une cinquantaine de pages, plusieurs portraits de « vies volées ». En parallèle avec l'actualité, plusieurs photos rappellent les discriminations entretenues par l'État envers les populations colonisées, le régime d'apartheid des ÉtatsUnis et les violences faites envers les Noirs.

La mort. Cette partie respecte la chronologie des mises à mort opérées par le régime nazi en publiant des photos de personnes condamnées car handicapées ou ces «vies 
sans valeur »! Elle poursuit en insistant sur la mort omniprésente dans les ghettos et sur les exécutions de masse qui ravagent les communautés juives d'Europe de l'est. Elle se focalise aussi sur la «mort industrielle » pratiquée dans les camps d'extermination. Les auteurs s'attardent sur la cruauté morbide du système concentrationnaire en associant des photos des gardiens des camps, heureux de vivre et insouciants de bonheur! Mais la mort concerne aussi les bourreaux. Le " châtiment » s'abat sur les dignitaires nazis que des photos montrent lors du procès de Nuremberg, comme il s'abat sur Eichman jugé à Jérusalem. En parallèle avec l'actualité, des clichés n'oublient pas les morts dus aux travaux forcés dans les colonies, en Afrique ou partout ailleurs où les droits humains sont bafoués.

Si les textes sont plutôt succincts, l'iconographie est très ample et très riche. Le catalogue donne beaucoup à voir s'il ne donne pas beaucoup à lire. Et c'était sans doute le but des auteurs d'une exposition. Il peut être assimilé à une encyclopédie visuelle même si certaines photos ont pu être remises en cause par la critique fine de Tal Bruttmann.

Danielle Delmaire

Perego Simon, Pleurons-les. Les Juifs de Paris et la commémoration de la Shoah (1944-1967), Champ-Vallon, «Époques », oct. 2020, 392 p., $26 €$, numérique $18 €$.

Tiré de sa thèse de doctorat soutenue en 2016, l'ouvrage de Simon Perego traite des commémorations de la Shoah par les organisations juives parisiennes de 1944, date de la libération de la capitale, à la guerre des Six Jours en 1967. L'un des intérêts principaux de ce livre est de retranscrire de manière très fine le foisonnement de ces rassemblements, attestant ainsi de la reconstruction et du dynamisme de la vie juive à Paris dès la fin de la guerre. L'originalité méthodologique réside dans la variété des angles d'analyse et le déplacement permanent des échelles d'observation: entre commémorateurs et public; entre individus et groupements ; entre public et privé ; entre espace et temps ; entre local et général.

Dans l'introduction, l'auteur situe de manière habile son travail par rapport à l'historiographie de la première mémoire de la Shoah d'une part, et à celle des Juifs de France dans la deuxième moitié du $\mathrm{XX}^{\mathrm{e}}$ siècle d'autre part. Il revient notamment sur les apports du livre Déportation et génocide d'Annette Wieviorka (1992) et sur la contestation du « mythe du silence » mettant en évidence la prise en compte précoce de la spécificité du sort subi. Outre l'analyse approfondie des rassemblements commémoratifs durant cette période, l'autre objectif est de proposer en parallèle une histoire des Juifs de Paris, un groupe présenté comme « restreint mais néanmoins dense et composite » (p. 21).

Divisé en trois parties, le livre aborde de façon thématique l'ensemble des enjeux de la commémoration. Justifié par la constance de l'activité commémorative observée durant la période étudiée, le choix d'un plan thématique impose néanmoins, en raison de la méthodologie adoptée, la reprise systématique de certains éléments (calendrier, cadres spatiaux-temporels, ritualisation, analyse discursive etc.) dans les différentes parties. Nous allons tenter de les passer en revue.

Si tous les courants de ce que l'auteur appelle «l'archipel juif de Paris» se sont appropriés le geste commémoratif, contribuant ainsi à renforcer l'image d'un groupe unifié et homogène formant une "communauté commémorative », la première partie révèle aussi comment la pratique de l'activité rituelle a été l'expression de la fragmentation de la judaïcité parisienne entre organisations religieuses, laïques, sionistes, bundistes, communistes; de ses conflictualités internes, exacerbées par le contexte de la Guerre froide; et de sa forte politisation. Simon Perego insiste avec 
raison sur les tensions politiques et idéologiques qui traversent cette collectivité. Quelques éléments comparatifs avec d'autres groupes juifs du territoire national auraient permis de mieux faire ressortir cette spécificité locale. Par ailleurs, l'étude de l'élaboration du calendrier commémoratif est l'occasion de rappeler la précocité des rassemblements honorant la mémoire des victimes du génocide. Ainsi, le premier de ces rassemblements a été organisé par l'association cultuelle israélite de Paris à partir de 1946 à la grande synagogue de la rue de la Victoire. Impliquant diverses organisations juives locales (communistes, sionistes, religieux, bundistes, etc.) d'autres cérémonies se déroulent dont certaines sont dédiées à des événements particuliers, tels que le soulèvement du ghetto de Varsovie, afin d'entretenir aussi la mémoire de la résistance des Juifs. Le choix des lieux devenus des sites commémoratifs comme la cité de la Muette à Drancy, le Vel' d'Hiv' ou le Mémorial du martyr juif inconnu permet à l'auteur d'appréhender de manière pertinente la question de la spatialité de cette pratique.

Adoptant une approche culturelle et religieuse de la mémoire, la deuxième partie porte sur les enjeux mémoriels revêtus par ces commémorations. Dans un premier temps, les cadres spatio-temporels et la ritualisation de l'hommage aux victimes ont été abordés. Simon Perego montre comment, dès l'immédiate après-guerre, le calendrier religieux a été mobilisé par les autorités consistoriales pour adopter une date spécifiquement dédiée à la commémoration de toutes les victimes juives. Fixée le dimanche précédant le nouvel an juif, « la cérémonie eut lieu pour la première fois le 22 septembre 1946 et fut reconduite chaque année jusqu'à aujourd'hui » (p. 140). La dimension spatiale de l'hommage révèle l'importance de ces lieux qui représentent, aux yeux des survivants et des endeuillés, « des tombes de substitution » (p. 147). Quant à la ritualisation, elle se caractérise par l'intégration d'éléments religieux (récitation de prières, usage des bougies) mais aussi par la place considérable accordée à la conservation des noms. Autre point important de cette deuxième partie, la fabrique mémorielle est appréhendée à travers l'étude des discours, de la terminologie employée, des événements commémorés et de leur interprétation. Si le sort spécifique des Juifs est affirmé très tôt, l'auteur observe aussi une «tentation de l'universalisation» (p. 177) et une appropriation de la vision globalisante des crimes nazis par certains acteurs de la scène commémorative juive à Paris. Enfin, l'analyse de l'enjeu de la transmission mémorielle met l'accent sur la volonté des organisateurs d'associer la jeunesse à ces manifestations. En ayant recours à des carnets personnels et/ou à des entretiens, le sujet aurait pu être aussi abordé du point de vue des enfants afin de questionner leurs représentations, leur engagement et leur réceptivité.

Les commémorations représentent aussi des moments particuliers où les appartenances identitaires peuvent s'affirmer publiquement. Dans cette dernière partie, trois pôles d'identification ont été retenus. Le premier est celui de la religion. L'auteur souligne la volonté des dirigeants consistoriaux d'ancrer les rassemblements dans la vie cultuelle. La commémoration devient même pour les plus éloignés de la religion le dernier lien avec une forme de sacralité. S'appuyant sur le cas du Mémorial du martyr juif inconnu, des pages très intéressantes sont consacrées aux rapports étroits et parfois conflictuels qu'ont pu entretenir religion et commémoration. L'appartenance française représente le deuxième pôle étudié. La participation des pouvoirs publics, des représentants des grands partis politiques et des «non-Juifs » revêt alors une dimension symbolique forte. Un monument commémoratif à l'intérieur de la grande synagogue de la rue de la Victoire a été inauguré le 27 février 1949 par le président de la République Vincent Auriol. Fait très intéressant, lors des discours, tous les sujets sont déjà évoqués, celui plus glorieux des «Justes » visant à valoriser l'engagement de Français dans le sauvetage de Juifs mais aussi celui encore très sensible de la complicité de la France et des responsabilités du régime de Vichy. En revanche, la réflexion sur l'utilisation de la 
catégorie des « non-Juifs » et la confusion possible entre religion et nationalité chez les organisateurs ou dans les articles de presse aurait pu être davantage approfondie. Enfin, la référence à Israël constitue le troisième et dernier pôle d'identification abordé. Les prises de parole lors des commémorations organisées dans les années d'après-guerre par les sionistes, les bundistes ou les communistes reflètent les divergences d'opinion qui existent alors au sein de la judaïcité parisienne à propos de l'État d'Israël. Des liens ont pu être établis par les orateurs entre la Shoah et les dangers encourus par le pays. Enfin, les dernières pages reviennent notamment sur l'existence d'une concurrence commémorative entre le projet de mémorial à Jérusalem et celui d'Isaac Schneersohn à Paris.

Il est toujours possible de discuter de la densité de l'ouvrage qui s'adresse prioritairement à des lecteurs avertis, de questionner le choix des limites chronologiques ou celui d'un cadre spatial trop parisiano-centré. Cependant, l'ouvrage de Simon Perego invite aussi à une véritable réflexion sur ces processus dans d'autres régions du territoire national à l'image de ce que nous avons modestement tenté de faire dans le dernier chapitre de notre thèse de doctorat consacrée aux logiques d'identification et d'appartenance des Juifs dans la zone littorale du Nord et du Pas-de-Calais. Surtout, les très nombreux apports historiographiques, la combinaison des points de vue mobilisés, l'utilisation d'une grande diversité de fonds d'archives, la collecte massive de données et la maitrise solide de la bibliographie reflètent la qualité scientifique de ce livre qui s'impose désormais comme une étude de référence sur la mémoire de la Shoah en France.

Rudy Rigaut

Horvilleur Delphine, Vivre avec nos morts, Paris, Grasset, 2021, 223 p., 19,50 €.

D'emblée arrêtons-nous sur le titre pour remarquer l'antinomie entre «vivre » et « morts » et retenir le possessif « nos ». Tout au long de l'ouvrage, la vie et la mort sont associées et le lecteur referme le livre avec ces derniers mots en hébreu:

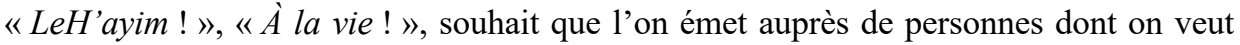
du bien. Il s'agit bien d'un livre sur la vie comme sur la mort car ce «LeH'ayim ! » ponctue les chapitres. Il s'agit aussi de vivre avec les morts auxquels nous sommes attachés, nos proches, nos amis, bref des intimes.

Onze chapitres composent l'ouvrage dans lesquels la rabbine revient sur ses expériences d'accompagnement de familles lors de funérailles, au bord de la tombe mais aussi au domicile du défunt ou encore en tout autre lieu, là où elle a été convoquée. Ce récit de ses expériences est l'occasion, maintes fois, de renseigner sur les coutumes et les rites funéraires juifs, sur les croyances des juifs et sur leur histoire. En d'autres pages, ce sont des mots hébreux, mots clés du judaïsme, qui sont expliqués avec des précisions sur leur sémantique et leur usage. De la sorte, l'ouvrage ne s'adresse pas aux initiés juifs mais il est abordable par tout lecteur ignorant du judaïsme. Les références à des textes rabbiniques, du Talmud ou de la Mishna ou encore du Shoulhan aroukh, la Loi juive codifiée au XVI ${ }^{\mathrm{e}}$ siècle par Joseph Karo, sont les bienvenues sans oublier les truculentes « histoires juives », salutaire détente.

Chacun des onze chapitres porte en titre le nom d'un défunt ou d'un proche du défunt. L'on peut répartir les témoignages en trois catégories : son soutien à des membres de la communauté que l'auteure encadre en tant que rabbine, sa présence auprès de personnalités qu'elle a côtoyées, son souvenir de personnages bibliques à propos d'un décès.

La première catégorie est la plus fournie. En diverses occasions, Delphine Horvilleur eut à aider des familles à réaliser un deuil. Tâche ardue et l'auteure partage avec le 
lecteur ses doutes, ses efforts et parfois ses maladresses dues à l'impuissance de consoler. « Trouver les mots et connaître ces gestes est le cœur de mon travail « (p. 16), reconnaît-elle. Il en va ainsi pour Azraël ( $1^{\mathrm{er}}$ chap.) ; Marc ( $3^{\mathrm{e}}$ chap) pour lequel elle ne souhaite pas dramatiser la mort, la séparation; Sarah (4e chap.), la cosette du ghetto dont la vie fut une longue et perpétuelle souffrance avoue son fils; le frère d'Isaac $\left(6^{\mathrm{e}}\right.$ chap.) qui exige de savoir où désormais se trouve son frère décédé : au ciel ou ailleurs, il doit savoir pour mieux vivre; Ariane ( $7^{\mathrm{e}}$ chap.) l'amie ou jumelle tant Delphine Horvilleur lui ressemble tout en étant si différente; et enfin Myriam ( $8^{\mathrm{e}}$ chap.) obsédée par ses funérailles auxquelles elle finit par assister de son vivant!

La seconde catégorie plus brève fait état de trois accompagnements : celui d'Elsa Cayat de Charlie Hebdo ( $2^{\mathrm{e}}$ chap.) dont elle partageait l'amitié malgré les différences d'implication dans le judaïsme ; celui de Marceline et Simone ( $5^{\mathrm{e}}$ chap.), inséparables, Delphine Horvilleur se trouve aux côtés de Marceline lors des obsèques de Simone et du transfert de ses cendres au Panthéon ce qui lui permet de restituer la gouaille de Marceline ; celui d'Isthaq Rabin ( $10^{\mathrm{e}}$ chap.) dont elle n'accompagne pas les funérailles mais elle relate les circonstances dans lesquelles elle vécut ce drame alors qu'elle-même vivait en Israël qu'elle a quitté par la suite.

La troisième catégorie est encore plus brève avec deux récits : celui de Moïse ( $9^{\mathrm{e}}$ chap.) « l'homme qui ne voulait pas mourir » qui donne l'occasion de relire le texte biblique et celui d'Edgard (11 $\mathrm{e}$ chap.), oncle de l'auteure dont le regard figé dans un tableau de famille scrute la petite fille qu'était l'auteure à la manière dont « l'œil » poursuit Caïn jusque dans la tombe, référence à Hugo.

Sollicitée toujours dans la précipitation puisque, dans le judaïsme, les funérailles suivent de très près le décès, la rabbine Delphine Horvilleur fait part de ses doutes, sans ostentation et souvent avec sincérité. Le lecteur partage très volontiers ses hésitations et peut la remercier de mieux faire connaître les rites funéraires juifs, le judaïsme en général et ses croyances en particulier. Un bel ouvrage à mettre entre les mains de quiconque souhaite connaître le métier de rabbin.

Danielle Delmaire 